\title{
BIOMARCADORES GLIAIS DA DOENÇA DE ALZHEIMER
}

\section{GLIAL BIOMARKERS OF ALZHEIMER'S DISEASE}

\author{
Matheus Fernandes Bittencourt ${ }^{1}$, Nathalia Vieira Müller ${ }^{1,2}$
}

\section{RESUMO}

Introdução: A neuroinflamação associada às células gliais é um elemento importante do processo patológico da doença de Alzheimer (DA). Este estudo apresenta uma revisão dos marcadores gliais quitinase 3-like 1 (YKL-40), do receptor desencadeado expresso nas células mieloides 2 (Triggering receptor expressed on myeloid cells 2 - TREM2), da proteína acídica fibrilar glial (GFAP) e da proteína B S100 ligante de cálcio (S100B). Métodos: Nesta revisão são analisados os marcadores gliais YKL-40, TREM2, GFAP e S100B presentes em sangue e/ou líquido cefalorraquidiano (LCR), a partir de estudos publicados até 2020 nos bancos de dados do PubMed, Medline e Periódicos Capes. Resultados: Foram recuperados 233 documentos, dentre os quais foram incluídos 60 . Todos os marcadores se encontram aumentados na DA em LCR - YKL-40 e TREM2 solúvel (sTREM2), já na fase pré-clínica -, e em sangue, e estão correlacionados ao declínio cognitivo. No entanto, nenhum dos marcadores analisados apresentou grande potencial para o diagnóstico diferencial. Além da proteína TREM2 solúvel no LCR, no sangue também se pode identificar alteração nos níveis do RNAm de TREM2. GFAP sanguíneo mostra ser o melhor em distinguir controles de pacientes com Alzheimer. Há evidências de um efeito protetivo da ativação glial em reação ao acúmulo amiloide. Conclusão: Os marcadores gliais no geral têm pouca utilidade para o diagnóstico diferencial, mas podem auxiliar no prognóstico e como biomarcadores inespecíficos para doenças neurodegenerativas.

Palavras-chave: Demência de Alzheimer; Glia; Biomarcador; Astrócitos

\section{ABSTRACT}

Introduction: Glial cell-associated neuroinflammation is a driving force for the pathological process of Alzheimer's disease (AD). This study is a systematic review aimed to analyze the following glial markers: chitinase-3-like protein 1 (YKL-40), triggering receptor expressed on myeloid cells 2 (TREM2), glial fibrillary acidic protein (GFAP) and S100 calcium-binding protein B (S100B). Methods: The PubMed, MEDLINE and CAPES Journals databases were searched for studies published until 2020 that addressed blood and/or cerebrospinal fluid (CSF) levels of YKL-40, TREM2, GFAP and S100B. Results: A total of 233 articles were retrieved, of which 60 were included in this study. All CSF - YKL-40 and soluble TREM2 (sTREM2) in preclinical stage - and blood biomarker levels were elevated for $A D$ and were correlated to cognitive decline. None of the analyzed biomarkers showed promising results for differential diagnosis. Besides CSF sTREM2 levels, blood TREM2 mRNA levels were also altered in AD. Blood GFAP levels seem to be the best option for distinguishing controls from AD patients.' There is evidence of a protective role of glial activation in amyloid accumulation. Conclusion: Glial markers in general are of little use for differential diagnosis but can assist in prognosis and as nonspecific biomarkers of neurodegenerative diseases.

Keywords: Alzheimer Dementia; Glia; Biomarker; Astrocytes

\section{INTRODUÇÃO}

A doença de Alzheimer (DA) é a mais frequente entre as demências, constituindo cerca de $60 \%$ a $70 \%$ de todos os $\operatorname{casos}^{1,2}$. A idade é o principal
Clin Biomed Res. 2021;41(2):157-166

1 Faculdade de Biomedicina, Universidade Católica de Petrópolis. Petrópolis, RJ, Brasil.

2 Laboratório de Microscopia Aplicada a Ciências da Vida, Instituto Nacional de Metrologia, Qualidade e Tecnologia. Duque de Caxias, RJ, Brasil.

Autor correspondente: Nathalia Vieira Müller nathaliavmuller@gmail.com Laboratório de Microscopia Aplicada a Ciências da Vida, Instituto Nacional de Metrologia, Qualidade e Tecnologia Av. Nossa Senhora das Graças, 50 25250-020, Duque de Caxias, RJ, Brasil. 
fator de risco, passando de $10 \%$ de prevalência na faixa etária de 65 anos para $40 \%$ em indivíduos acima de 80 anos. A DA é uma desordem crônica e progressiva relacionada com a destruição dos neurônicos colinérgicos ${ }^{3}$. A patogênese da DA se baseia nos dois processos observados por Alois Alzheimer em 1906, a deposição de $\beta$ amiloide $(A \beta)$ das placas senis e a de tau nos emaranhados neurofibrilares $(E N F)^{4}$. $\mathrm{O} A \beta$ de 42 aminoácidos (A $\beta 42$ ) fluido, juntamente com as concentrações total e fosforilada de tau (t-tau e p-tau respectivamente), são biomarcadores aceitos para suportar o diagnóstico de DA, refletindo as placas senis e os ENF ${ }^{5}$. A neuroinflamação vem sendo considerada como um fator que impulsiona processo patológico da $D A$, já que as placas $A \beta$ e os emaranhados induzem uma resposta imunológica no cérebro, caracterizada pela ativação das células da glia ${ }^{5}$. Entretanto, não se é completamente compreendido se a neuroinflamação representa um efeito danoso ou um mecanismo compensatório, ou até ambos, em questão ao acúmulo amiloide, neurodegeneração e função cognitiva ${ }^{6}$. Micróglia e astrócitos são os principais tipos de células gliais associadas a DA. Os astrócitos são as células gliais de maior abundância, e tem importante função na homeostase, transmissão de sinais, sinaptogênese, plasticidade sináptica e suporte metabólico para os neurônios ${ }^{5}$. Micróglia representa as células de linhagem mieloide presentes no sistema nervoso central (SNC), e atuam na resposta imunológica assim como na homeostase cerebral, e em conjunto com astrócitos podem desenvolver propriedades inflamatórias ${ }^{7}$. Tanto micróglia quanto astrócitos ativados estão colocalizados com as placas senis e emaranhados neurofibrilares em cérebro de pacientes de $\mathrm{DA}^{8}$. Os quatro candidatos a biomarcadores aqui estudados são: quitinase 3-like 1 (YKL-40), Receptor desencadeado expresso nas células mielóides 2 (Triggering receptor expressed on myeloid cells 2 - TREM2), proteína acídica fibrilar glial (GFAP) e proteína B S100 ligante de cálcio (S100B). Esse estudo tem como objetivo avaliar quais os benefícios, em questão de diagnóstico e prognóstico, os indivíduos no espectro da doença de Alzheimer, poderiam obter com a avaliação dos biomarcadores gliais YKL-40, TREM2, GFAP e S100B em seu sangue ou LCR.

\section{MÉTODOS}

Essa revisão sistemática foi feita a partir de busca nos bancos de dados do PubMed, MEDLINE e
Periódicos CAPES, utilizando a combinação de descritores: "Alzheimer" e "biomarker", em conjunto com os biomarcadores "YKL-40", "TREM2", "GFAP" e "S100B". A busca no PubMed foi feita com filtros para resultados com todos os termos no título ou resumo (Title/Abstract), para Periódicos CAPES, os termos 'alzheimer biomarker' poderiam estar presentes em qualquer local do texto, mas o nome do marcador deveria estar 'no assunto', e em MEDLINE não foram usados filtros, mas foi selecionada a opção 'todas as palavras' (marcador booleano 'AND'). Como critérios de inclusão, os trabalhos deveriam envolver ensaios clínicos que registraram a utilização de biomarcadores fluidos para a doença de Alzheimer e deveriam ter sido publicados até $31 / 12 / 2020$. Como critérios de exclusão foram considerados trabalhos de revisão, os que utilizavam somente modelo animal e/ou in vitro, os que tinham foco em doenças que não a de Alzheimer, os que não investigassem as moléculas em LCR e/ou plasma ou quanto a sua função como biomarcadores.

As ferramentas utilizadas para a avaliação de qualidade dos estudos foram os checklists do Joanna Briggs Institute (JBI), compostos por perguntas cujas respostas seriam 'sim', 'não', 'incerto' ou 'não se aplica' (NA), e as perguntas são elaboradas de forma que a resposta "sim" represente um ponto favorável a qualidade e menor risco de viés. Foram usados os modelos de coorte, teste de acurácia diagnóstica, caso-controle e de estudos transversais, o que fosse mais adequado ao desenho de cada estudo.

\section{RESULTADO E DISCUSSÃO}

A partir dos descritores usados, foram recuperados um total de 304 documentos, 81 de TREM2 (11 de MEDLINE, 39 de PubMed e 31 de Periódicos CAPES), 110 de YKL-40 (26 de MEDLINE, 60 de PubMed e 24 de Periódicos CAPES), 71 de GFAP (22 de MEDLINE, 32 de PubMed e 27 de Periódicos CAPES) e 81 de S100B (12 de MEDLINE, 16 de PubMed e 53 de Periódicos CAPES). Após unificação dos bancos de dados e eliminação de duplicatas $r$ estaram 54 de TREM2, 71 de YKL-40, 61 de GFAP e 67 de S100B. Eliminando as duplicatas entre os grupos de marcadores, restaram 233 artigos. Durante o processo de triagem com base em título e resumo foram eliminados 158 artigos totalizando 75 restantes. Após a leitura completa, foram eliminados mais 16 artigos, restando 59 na seleção final. A Tabela 1 compila a avaliação dos estudos. 
Tabela 1: Avaliação de qualidade dos estudos.

\begin{tabular}{|c|c|c|c|c|c|c|c|c|c|c|c|c|c|c|}
\hline & \multirow{2}{*}{ Autor } & \multirow{2}{*}{ Modelo } & \multicolumn{11}{|c|}{ Critérios } & \multirow{2}{*}{$\begin{array}{l}\text { Sim } \\
(\%)\end{array}$} \\
\hline & & & 1 & 2 & 3 & 4 & 5 & 6 & 7 & 8 & 9 & 10 & 11 & \\
\hline 1 & $\begin{array}{l}\text { Vergallo } \\
\text { et al. }{ }^{6}\end{array}$ & Coorte & Sim & Sim & Sim & Sim & Sim & Sim & Sim & Não & Sim & NA & Sim & $90 \%$ \\
\hline 2 & $\begin{array}{l}\text { Sutphen } \\
\text { et al. }{ }^{9}\end{array}$ & Coorte & Sim & Sim & Sim & Sim & Sim & Sim & Sim & Não & Sim & NA & Sim & $90 \%$ \\
\hline 3 & $\begin{array}{l}\text { Craig- } \\
\text { Schapiro } \\
\text { et al. }^{10}\end{array}$ & Coorte & Sim & Sim & Sim & Sim & Sim & Sim & Sim & Não & Incerto & NA & Sim & $80 \%$ \\
\hline $\begin{array}{l}\text { Sim } \\
(\%)\end{array}$ & & & $100 \%$ & $100 \%$ & $100 \%$ & $100 \%$ & $100 \%$ & $100 \%$ & $100 \%$ & $0 \%$ & $67 \%$ & $0 \%$ & $100 \%$ & \\
\hline 1 & $\begin{array}{l}\text { Paterson } \\
\text { et al. }{ }^{11}\end{array}$ & $\begin{array}{l}\text { Acurácia } \\
\text { Diagnóstica }\end{array}$ & Incerto & Sim & Sim & Incerto & NA & Sim & Incerto & Sim & Sim & Não & & $50 \%$ \\
\hline 2 & $\begin{array}{l}\text { Hampel } \\
\text { et al. }{ }^{12}\end{array}$ & $\begin{array}{l}\text { Acurácia } \\
\text { Diagnóstica }\end{array}$ & Incerto & Não & Não & Incerto & Sim & Sim & Incerto & Incerto & Sim & NA & & $30 \%$ \\
\hline 3 & $\begin{array}{l}\text { Alcolea } \\
\text { et al. } .^{13}\end{array}$ & $\begin{array}{l}\text { Acurácia } \\
\text { Diagnóstica }\end{array}$ & Incerto & Não & Sim & Não & NA & Sim & Sim & Sim & Sim & Sim & & $60 \%$ \\
\hline 4 & $\begin{array}{l}\text { Paterson } \\
\text { et al. }{ }^{14}\end{array}$ & $\begin{array}{l}\text { Acurácia } \\
\text { Diagnóstica }\end{array}$ & Sim & Não & Sim & Incerto & NA & Sim & Incerto & Sim & Sim & Sim & & $60 \%$ \\
\hline 5 & $\begin{array}{l}\text { Mattsson } \\
\text { et al. }{ }^{15}\end{array}$ & $\begin{array}{l}\text { Acurácia } \\
\text { Diagnóstica }\end{array}$ & Sim & Não & Sim & Incerto & NA & Sim & Incerto & Sim & Sim & Não & & $50 \%$ \\
\hline 6 & $\begin{array}{l}\text { Antonell } \\
\text { et al. }{ }^{16}\end{array}$ & $\begin{array}{l}\text { Acurácia } \\
\text { Diagnóstica }\end{array}$ & Incerto & Não & Sim & Não & Não & Sim & Incerto & Sim & Sim & Sim & & $50 \%$ \\
\hline 7 & $\begin{array}{l}\text { Baldacci } \\
\text { et al. }{ }^{17}\end{array}$ & $\begin{array}{l}\text { Acurácia } \\
\text { Diagnóstica }\end{array}$ & Não & Não & Sim & Não & NA & Sim & Incerto & Sim & Sim & Sim & & $50 \%$ \\
\hline $\begin{array}{l}\text { Sim } \\
(\%)\end{array}$ & & & $29 \%$ & $14 \%$ & $86 \%$ & $0 \%$ & $14 \%$ & $100 \%$ & $14 \%$ & $86 \%$ & $100 \%$ & $57 \%$ & & \\
\hline 1 & $\begin{array}{l}\text { Van Hulle } \\
\text { et al. }{ }^{18}\end{array}$ & $\begin{array}{l}\text { Caso- } \\
\text { Controle }\end{array}$ & Sim & Incerto & Sim & Sim & Sim & Sim & Sim & Sim & NA & Sim & & $80 \%$ \\
\hline 2 & $\begin{array}{l}\text { Gispert } \\
\text { et al. }{ }^{19}\end{array}$ & $\begin{array}{l}\text { Caso- } \\
\text { Controle }\end{array}$ & Sim & Incerto & Sim & Sim & Sim & Sim & Sim & Sim & NA & Sim & & $80 \%$ \\
\hline 3 & $\begin{array}{l}\text { Abu- } \\
\text { Rumeileh } \\
\text { et al. }{ }^{20}\end{array}$ & $\begin{array}{l}\text { Caso- } \\
\text { Controle }\end{array}$ & Sim & Sim & Sim & Sim & Sim & Sim & Sim & Sim & NA & Sim & & $90 \%$ \\
\hline 4 & $\begin{array}{l}\text { Rauchmann } \\
\text { et al. }{ }^{21}\end{array}$ & $\begin{array}{l}\text { Caso- } \\
\text { Controle }\end{array}$ & Sim & Incerto & Sim & Sim & Sim & Sim & Sim & Sim & NA & Sim & & $80 \%$ \\
\hline 5 & $\begin{array}{l}\text { Lauridsen } \\
\text { et al. }{ }^{22}\end{array}$ & $\begin{array}{l}\text { Caso- } \\
\text { Controle }\end{array}$ & Sim & Sim & Sim & Sim & Sim & Sim & Sim & Sim & NA & Sim & & $90 \%$ \\
\hline 6 & $\begin{array}{l}\text { Banerjee } \\
\text { et al. }{ }^{23}\end{array}$ & $\begin{array}{l}\text { Caso- } \\
\text { Controle }\end{array}$ & Sim & Sim & Sim & Sim & Sim & Sim & Sim & Sim & NA & Sim & & $90 \%$ \\
\hline 7 & $\begin{array}{l}\text { Llorens } \\
\text { et al. }{ }^{24}\end{array}$ & $\begin{array}{l}\text { Caso- } \\
\text { Controle }\end{array}$ & Sim & Incerto & Sim & Sim & Sim & Não & Incerto & Sim & NA & Sim & & $60 \%$ \\
\hline 8 & Bos et al. ${ }^{25}$ & $\begin{array}{l}\text { Caso- } \\
\text { Controle }\end{array}$ & Sim & Incerto & Sim & Sim & Sim & Sim & Sim & Sim & NA & Sim & & $80 \%$ \\
\hline 9 & $\begin{array}{l}\text { Knapskog } \\
\text { et al. }{ }^{26}\end{array}$ & $\begin{array}{l}\text { Caso- } \\
\text { Controle }\end{array}$ & Sim & Sim & Sim & Sim & Sim & Sim & Sim & Sim & NA & Sim & & $90 \%$ \\
\hline 10 & $\begin{array}{l}\text { Henjum } \\
\text { et al. }{ }^{27}\end{array}$ & $\begin{array}{l}\text { Caso- } \\
\text { Controle }\end{array}$ & Sim & Sim & Sim & Sim & Sim & Não & Incerto & Sim & NA & Sim & & $70 \%$ \\
\hline 11 & $\begin{array}{l}\text { Brosseron } \\
\text { et al. }{ }^{28}\end{array}$ & $\begin{array}{l}\text { Caso- } \\
\text { Controle }\end{array}$ & Sim & Incerto & Sim & Sim & Sim & Sim & Sim & Sim & NA & Sim & & $80 \%$ \\
\hline 12 & $\begin{array}{l}\text { Deming } \\
\text { et al. }{ }^{29}\end{array}$ & $\begin{array}{l}\text { Caso- } \\
\text { Controle }\end{array}$ & Sim & Incerto & Sim & Sim & Sim & Sim & Sim & Sim & NA & Sim & & $80 \%$ \\
\hline 13 & $\begin{array}{l}\text { Ozaki } \\
\text { et al. }{ }^{30}\end{array}$ & $\begin{array}{l}\text { Caso- } \\
\text { Controle }\end{array}$ & Sim & Sim & Sim & Sim & Sim & Sim & Sim & Sim & NA & Sim & & $90 \%$ \\
\hline
\end{tabular}


Tabela 1: Continuação

\begin{tabular}{|c|c|c|c|c|c|c|c|c|c|c|c|c|c|c|}
\hline & \multirow{2}{*}{ Autor } & \multirow{2}{*}{ Modelo } & \multicolumn{11}{|c|}{ Critérios } & \multirow{2}{*}{$\begin{array}{l}\text { Sim } \\
(\%)\end{array}$} \\
\hline & & & 1 & 2 & 3 & 4 & 5 & 6 & 7 & 8 & 9 & 10 & 11 & \\
\hline 14 & $\begin{array}{l}\text { Morenas- } \\
\text { rodríguez } \\
\text { et al. }{ }^{31}\end{array}$ & $\begin{array}{l}\text { Caso- } \\
\text { Controle }\end{array}$ & Sim & Não & Sim & Sim & Sim & Sim & Sim & Sim & NA & Sim & & $80 \%$ \\
\hline 15 & $\begin{array}{l}\text { Gruden } \\
\text { et al. } .^{32}\end{array}$ & $\begin{array}{l}\text { Caso- } \\
\text { Controle }\end{array}$ & Sim & Sim & Sim & Sim & Sim & Não & Não & Sim & NA & Não & & $60 \%$ \\
\hline 16 & Ma et al. ${ }^{33}$ & $\begin{array}{l}\text { Caso- } \\
\text { Controle }\end{array}$ & Sim & Incerto & Sim & Sim & Sim & Sim & Sim & Sim & NA & Sim & & $80 \%$ \\
\hline 17 & $\begin{array}{l}\text { Suárez- } \\
\text { calvet } \\
\text { et al. }{ }^{34}\end{array}$ & $\begin{array}{l}\text { Caso- } \\
\text { Controle }\end{array}$ & Sim & Incerto & Sim & Sim & Sim & Sim & Sim & Sim & NA & Sim & & $80 \%$ \\
\hline 18 & Liu et al. ${ }^{35}$ & $\begin{array}{l}\text { Caso- } \\
\text { Controle }\end{array}$ & Sim & Incerto & Sim & Sim & Sim & Sim & Sim & Sim & NA & Sim & & $80 \%$ \\
\hline 19 & Hou et al. ${ }^{36}$ & $\begin{array}{l}\text { Caso- } \\
\text { Controle }\end{array}$ & Sim & Sim & Sim & Sim & Sim & Sim & Sim & Sim & NA & Sim & & $90 \%$ \\
\hline 20 & $\begin{array}{l}\text { Oeckl } \\
\text { et al. }{ }^{37}\end{array}$ & $\begin{array}{l}\text { Caso- } \\
\text { Controle }\end{array}$ & Sim & Incerto & Sim & Sim & Sim & Sim & Sim & Sim & NA & Sim & & $80 \%$ \\
\hline 21 & $\begin{array}{l}\text { Nordengen } \\
\text { et al. }{ }^{7}\end{array}$ & $\begin{array}{l}\text { Caso- } \\
\text { Controle }\end{array}$ & Sim & Incerto & Sim & Sim & Sim & Sim & Sim & Sim & NA & Sim & & $80 \%$ \\
\hline 22 & $\begin{array}{l}\text { Ishiki } \\
\text { et al. }{ }^{38}\end{array}$ & $\begin{array}{l}\text { Caso- } \\
\text { Controle }\end{array}$ & Sim & Sim & Sim & Sim & Sim & Sim & Não & Sim & NA & Sim & & $80 \%$ \\
\hline 23 & $\begin{array}{l}\text { Ewers } \\
\text { et al. }{ }^{39}\end{array}$ & $\begin{array}{l}\text { Caso- } \\
\text { Controle }\end{array}$ & Sim & Sim & Sim & Sim & Sim & Sim & Sim & Sim & NA & Sim & & $90 \%$ \\
\hline 24 & Tan et al. ${ }^{40}$ & $\begin{array}{l}\text { Caso- } \\
\text { Controle }\end{array}$ & Sim & Sim & Sim & Sim & Sim & Sim & Sim & Sim & NA & Sim & & $90 \%$ \\
\hline 25 & $\begin{array}{l}\text { Heslegrave } \\
\text { et al. }{ }^{41}\end{array}$ & $\begin{array}{l}\text { Caso- } \\
\text { Controle }\end{array}$ & Sim & Sim & Sim & Sim & Sim & Não & Não & Sim & NA & Não & & $60 \%$ \\
\hline 26 & Hu et al. ${ }^{42}$ & $\begin{array}{l}\text { Caso- } \\
\text { Controle }\end{array}$ & Sim & Sim & Sim & Sim & Sim & Não & Não & Sim & NA & Não & & $60 \%$ \\
\hline 27 & $\begin{array}{l}\text { Casati } \\
\text { et al. } .^{43}\end{array}$ & $\begin{array}{l}\text { Caso- } \\
\text { Controle }\end{array}$ & Sim & Sim & Sim & Sim & Sim & Não & Não & Sim & NA & Não & & $60 \%$ \\
\hline 28 & Lleó et al. ${ }^{44}$ & $\begin{array}{l}\text { Caso- } \\
\text { Controle }\end{array}$ & Sim & Sim & Sim & Sim & Sim & Sim & Sim & Sim & NA & Sim & & $90 \%$ \\
\hline 29 & $\begin{array}{l}\text { Sutphen } \\
\text { et al. }{ }^{45}\end{array}$ & $\begin{array}{l}\text { Caso- } \\
\text { Controle }\end{array}$ & Sim & Sim & Sim & Sim & Sim & Sim & Sim & Sim & NA & Sim & & $90 \%$ \\
\hline 30 & $\begin{array}{l}\text { Merluzzi } \\
\text { et al. }{ }^{46}\end{array}$ & $\begin{array}{l}\text { Caso- } \\
\text { Controle }\end{array}$ & Sim & Sim & Sim & Sim & Sim & Sim & Sim & Sim & NA & Sim & & $90 \%$ \\
\hline 31 & Han et al. ${ }^{47}$ & $\begin{array}{l}\text { Caso- } \\
\text { Controle }\end{array}$ & Sim & Incerto & Sim & Sim & Sim & Não & Não & Sim & NA & Sim & & $60 \%$ \\
\hline 32 & $\begin{array}{l}\text { Asken } \\
\text { et al. }{ }^{48}\end{array}$ & $\begin{array}{l}\text { Caso- } \\
\text { Controle }\end{array}$ & Sim & Incerto & Sim & Sim & Sim & Sim & Sim & Sim & NA & Sim & & $80 \%$ \\
\hline 33 & $\begin{array}{l}\text { Villar- } \\
\text { Piqué } \\
\text { et al. }{ }^{49}\end{array}$ & $\begin{array}{l}\text { Caso- } \\
\text { Controle }\end{array}$ & Sim & Sim & Sim & Sim & Sim & Sim & Sim & Sim & NA & Sim & & $90 \%$ \\
\hline 34 & Choi et al. ${ }^{50}$ & $\begin{array}{l}\text { Caso- } \\
\text { Controle }\end{array}$ & Sim & Incerto & Sim & Sim & Sim & Sim & Sim & Sim & NA & Sim & & $80 \%$ \\
\hline 35 & $\begin{array}{l}\text { Morenas- } \\
\text { Rodríguez } \\
\text { et al. }{ }^{51}\end{array}$ & $\begin{array}{l}\text { Caso- } \\
\text { Controle }\end{array}$ & Não & Não & Sim & Sim & Sim & Sim & Sim & Sim & NA & Sim & & $70 \%$ \\
\hline 36 & $\begin{array}{l}\text { Sathe } \\
\text { et al. }{ }^{.2}\end{array}$ & $\begin{array}{l}\text { Caso- } \\
\text { Controle }\end{array}$ & Sim & Sim & Sim & Sim & Sim & Sim & Sim & Sim & NA & Sim & & $90 \%$ \\
\hline 37 & $\begin{array}{l}\text { Benussi } \\
\text { et al. }{ }^{53}\end{array}$ & $\begin{array}{l}\text { Caso- } \\
\text { Controle }\end{array}$ & Sim & Incerto & Sim & Sim & Sim & Sim & Não & Sim & NA & Sim & & $70 \%$ \\
\hline 38 & $\begin{array}{l}\text { Rauchmann } \\
\text { et al. }{ }^{54}\end{array}$ & $\begin{array}{l}\text { Caso- } \\
\text { Controle }\end{array}$ & Sim & Sim & Sim & Sim & Sim & Sim & Sim & Sim & NA & Sim & & $90 \%$ \\
\hline
\end{tabular}


Tabela 1: Continuação

\begin{tabular}{|c|c|c|c|c|c|c|c|c|c|c|c|c|c|c|}
\hline & \multirow{2}{*}{ Autor } & \multirow{2}{*}{ Modelo } & \multicolumn{11}{|c|}{ Critérios } & \multirow{2}{*}{$\begin{array}{l}\text { Sim } \\
(\%)\end{array}$} \\
\hline & & & 1 & 2 & 3 & 4 & 5 & 6 & 7 & 8 & 9 & 10 & 11 & \\
\hline 39 & Mori et al. ${ }^{55}$ & $\begin{array}{l}\text { Caso- } \\
\text { Controle }\end{array}$ & Sim & Sim & Sim & Sim & Sim & Não & Não & Sim & NA & Sim & & $70 \%$ \\
\hline 40 & $\begin{array}{l}\text { Gispert } \\
\text { et al. }{ }^{56}\end{array}$ & $\begin{array}{l}\text { Caso- } \\
\text { Controle }\end{array}$ & Sim & Sim & Sim & Sim & Sim & Sim & Sim & Sim & NA & Sim & & $90 \%$ \\
\hline 41 & $\begin{array}{l}\text { Llorens } \\
\text { et al. } .^{57}\end{array}$ & $\begin{array}{l}\text { Caso- } \\
\text { Controle }\end{array}$ & Sim & Incerto & Sim & Sim & Sim & Não & Não & Sim & NA & Sim & & $60 \%$ \\
\hline 42 & $\begin{array}{l}\text { Suárez- } \\
\text { Calvet } \\
\text { et al. }^{58}\end{array}$ & $\begin{array}{l}\text { Caso- } \\
\text { Controle }\end{array}$ & Sim & Sim & Sim & Sim & Sim & Sim & Sim & Sim & NA & Sim & & $90 \%$ \\
\hline $\begin{array}{l}\text { Sim } \\
(\%)\end{array}$ & & & $98 \%$ & $55 \%$ & $100 \%$ & $100 \%$ & $100 \%$ & $79 \%$ & $74 \%$ & $100 \%$ & $0 \%$ & $90 \%$ & & \\
\hline 1 & $\begin{array}{l}\text { Milà- } \\
\text { Alomà } \\
\text { et al. }^{59}\end{array}$ & Transversal & Sim & Sim & Sim & Sim & Sim & Sim & Sim & Sim & & & & $80 \%$ \\
\hline 2 & $\begin{array}{l}\text { Alcolea } \\
\text { et al. }{ }^{60}\end{array}$ & Transversal & Sim & Sim & Sim & Sim & Sim & Sim & Sim & Sim & & & & $80 \%$ \\
\hline 3 & $\begin{array}{l}\text { Teitsdottir } \\
\text { et al. }{ }^{61}\end{array}$ & Transversal & Sim & Sim & Sim & Sim & Sim & Sim & Sim & Sim & & & & $80 \%$ \\
\hline 4 & $\begin{array}{l}\text { Suárez- } \\
\text { Calvet } \\
\text { et al. }{ }^{62}\end{array}$ & Transversal & Sim & Não & Sim & Sim & Sim & Sim & Sim & Sim & & & & $70 \%$ \\
\hline 5 & $\begin{array}{l}\text { Palmqvist } \\
\text { et al. } .^{63}\end{array}$ & Transversal & Sim & Sim & Sim & Sim & Não & Incerto & Sim & Sim & & & & $60 \%$ \\
\hline 6 & $\begin{array}{l}\text { Wang } \\
\text { et al. }{ }^{64}\end{array}$ & Transversal & Sim & Não & Sim & Sim & Não & Incerto & Sim & Sim & & & & $50 \%$ \\
\hline 7 & $\begin{array}{l}\text { Höglund } \\
\text { et al. } 65\end{array}$ & Transversal & Sim & Sim & Sim & Sim & Não & Não & Sim & Sim & & & & $60 \%$ \\
\hline $\begin{array}{l}\text { Sim } \\
(\%)\end{array}$ & & & $100 \%$ & $71 \%$ & $100 \%$ & $100 \%$ & $57 \%$ & $57 \%$ & $100 \%$ & $100 \%$ & & & & \\
\hline
\end{tabular}

\section{YKL-40}

A quitinase 3-like 1, também conhecida como YKL-40, é uma glicoproteína de 40kDa que pertence à família das quitinases humanas e codificada pelo gene CHI3L1. Entretanto, a YKL-40 não tem a atividade de quitinase, tendo sua função biológica ainda indeterminada. A YKL-40 já foi demonstrada ter um papel na resposta de astrócitos para modular a neuroinflamação, porém não é muito bem compreendido. Além de astrócitos, a YKL-40 também é expressa em células da micróglia, em macrófagos perivasculares e em raros neurônios de substância branca ${ }^{19}$.

A YKL-40 pode ser mensurada no $L C R$ em pacientes de DA já na fase pré-clínica ${ }^{25}$, na fase prodrômica apresentando comprometimento cognitivo leve (CCL) e na fase demencial ${ }^{7,9,12,14,46,50,51,64,65}$, pouco depois de $A \beta 42$ e tau ${ }^{63}$. Esse aumento, contudo, não foi visto no estudo de Mattsson et al. ${ }^{15}$. Na DA, os níveis de YKL40 no LCR se relacionam a idade e aos níveis de tau fosforilada ( $p$-tau) e tau total (t-tau) no LCR, mas não com $A \beta 42^{6,9-11,13,22,29,60,61,64}$. Existe também a correlação da YKL-40 com a neurodegeneração associada a tau $^{19}$. Foi demonstrado aumento longitudinal em CCL com positividade $A \beta$, enquanto se via pouca variação no grupo $A \beta$ negativo ${ }^{44}$. Em indivíduos com CCL e com demência com perfil positivo DA (definido pela razão $p$-tau/Aß42), os níveis de YKL-40 mostraram-se mais elevados em relação aos com perfil negativo ${ }^{18}$. Gispert et al. ${ }^{56}$ e Wang et al. ${ }^{64}$ demonstraram o aumento das concentrações de YKL-40 em LCR em pacientes com CCL portadores do alelo APOE $\varepsilon 4$ (considerado fator de risco genético para a DA esporádica), em relação a pacientes não portadores com CCL, indicando uma ativação glial aumentada nos portadores. Outros estudos já não observaram associação entre os níveis de YKL-40 e APOE $\varepsilon 4^{6,25,44}$. O estudo de Deming et al. ${ }^{29}$ demonstrou que a variante rs10399931 do gene CHI3L1 influencia as concentrações de YKL-40 quase tanto quanto idade e gênero combinados, porém não foi associada ao maior risco de DA, idade de início ou progressão da doença. Vergallo et al. ${ }^{6}$ encontrou uma associação negativa entre os níveis de plasmáticos de YKL-40 e o acúmulo amiloide, ao mesmo tempo que verificou nenhuma associação entre o marcador e atividade neuronal, volume cerebral ou performance cognitiva, 
evidencias que suportam a hipótese de que a glia atua de forma compensatória durante a amiloidose cerebral na DA.

O estudo de Abu-Rumeileh et al..$^{20}$ demonstrou uma acurácia moderada da YKL-40 do LCR, com $\geq 80 \%$ de sensibilidade e especificidade em discriminar a DA de controles, e tendo demonstrado o valor da área sob a curva (AUC) de característica de operação do receptor igual a 0,882 , sendo um AUC de valor 1 o melhor possível. Já Llorens et al. ${ }^{24}$, Paterson et al. ${ }^{11}$ e Baldacci et al. ${ }^{17}$ tiveram resultados mais baixos de AUC, com 0,77, 0,75 e 0,69 respectivamente. O estudo de Antonell et al. ${ }^{16}$ demonstrou baixa capacidade de predição para YKL-40, com um AUC de somente 0,69, e não foi possível estabelecer um valor de corte entre os controles saudáveis e os pacientes de DA devido à alta variabilidade dos níveis do biomarcador nos controles. No trabalho de Teitsdottir et al. ${ }^{61}$, YKL-40 em LCR apresentou baixa capacidade de distinção $(A \cup C=0,64)$ entre indivíduos com perfil de DA e não-DA determinado pela razão t-tau/A $\beta 42$. Llorens et al. ${ }^{24}$ demonstrou também que YKL-40 em LCR não tem boa capacidade de distinção para DA e doença de Creutzfeldt-Jakob (AUC $=0,70$ ). O biomarcador no LCR se mostrou falho em diferenciar a DA de demência vascular, não apresentando diferença significante em seus níveis ${ }^{57}$.

Comparando os níveis de YKL-40 do LCR em pacientes com DA e demência frontotemporal (DFT), foi demonstrado que a concentração do marcador é mais elevada em $\mathrm{DA}^{12}$, e tendo demonstrado um AUC de 0,71 para diferenciar as demências no estudo de Baldacci et al. ${ }^{17}$. Porém, Abu-Rumeileh et al. ${ }^{20}$ demonstrou uma elevação em DA que não considerou significativamente diferente de DFT ao utilizar o mesmo kit ELISA (R\&D Systems), e Antonell et al. ${ }^{16}$ não observou diferença utilizando o kit ELISA da QUIDEL. O estudo de Morenas-Rodríguez et al. ${ }^{31}$ comparando a ativação glial em DA e a doença de corpo de Lewy (DCL) demonstrou que na fase prodrômica se verifica o aumento de YKL-40 em LCR somente na DA, e não há elevação de YKL-40 na fase demencial na DCL a não ser que haja patologia de DA concomitantemente.

Assim como em LCR, a YKL-40 plasmática está associada a idade ${ }^{49}$. No estudo de Villar-Piqué et al. ${ }^{49}$, foi detectado um aumento nos níveis da YKL-40 plasmática, porém, esse aumento não foi considerado estatisticamente significante se comparado aos controles ou outras doenças neurodegenerativas, e apresentando um AUC de somente 0,62 para diferenciar indivíduos com DA do grupo controle, além de ter um potencial incerto voltado ao diagnóstico diferencial no contexto de doenças neurodegenerativas, já que muitas dessas doenças apresentam elevação nos níveis da YKL-40 plasmática, incluindo a doença de Creutzfeldt-Jakob, DFT, demência vascular e DCL ${ }^{49}$.

\section{TREM2}

O TREM2 é um receptor do sistema imune inato da família das imunoglobulinas, e são expressos nas células da linhagem monocítica. No SNC esse receptor é expresso exclusivamente na micróglia e tem papel na fagocitose de bactérias e material apoptótico ${ }^{35}$. O gene Trem2 se localiza no cromossomo 6 e portadores da sua variante $\mathrm{R} 47 \mathrm{H}$ têm maior risco de desenvolver a DA. TREM2 é uma proteína transmembrana tipo 1 , com seu ectodomínio sendo clivado na membrana celular pela clivagem pelo ADAM10 ou ADAM17, o que produz o fragmento de proteína TREM2 solúvel (sTREM2), liberado no espaço extracelular e podendo ser encontrado no LCR e sangue ${ }^{35}$.

sTREM2 tem seu nível elevado já na fase présintomática, e foi visto aumento de 18\%-23\% em indivíduos em estado demencial ${ }^{41}$, porém aparenta ter seu pico na fase prodrômica, o que seria uma limitação para seu uso, complicando o desenvolvimento de um ponto de corte clínico ${ }^{31,58}$. Uma forte associação foi observada entre sTREM2 e a idade, patologia tau, YKL-40 e injuria neuronal, mas pouca ou nenhuma com níveis de $A \beta^{21,26,28,35,41,58}$. No estudo de Henjum et al. ${ }^{27}$, entretanto, só foi vista a correlação entre sTREM2, t-tau e p-tau em LCR no grupo de controles saudáveis, e não nos grupos com CCL ou DA, usando um novo ELISA foi desenvolvido para esse estudo. Morenas-Rodríguez et al. ${ }^{31}$ descreveu maior correlação entre sTREM2 e t-tau, p-tau e Aß42 em DA prodrômica em comparação a demencial, e no grupo controle a correlação entre sTREM2 e p-tau foi ainda maior. Foram demonstrados menores níveis de STREM2 associados a positividade $A \beta$, encontrandose abaixo dos controles na ausência de sinais de neurodegeneração ou patologia tau, e havendo as maiores elevações nos grupos $A \beta$-negativos, porém, onde os últimos se encontravam presentes ${ }^{33,34,54}$. O estudo de Ewers et al. ${ }^{39}$ demonstrou que altos níveis base de sTREM2 estavam associados a taxas mais lentas de acúmulo amiloide, suportando a hipótese de que sTREM2 e a atividade microglial tem efeito protetivo no DA. Uma possível explicação para esse fenômeno é a de que a micróglia inicialmente forma uma barreira entorno das placas senis, fazendo com que o sTREM2 liberado fique retido dentro da placa até que a barreira falhe e comece o dano neuronal subsequente ${ }^{33,34}$. Outro argumento é o de que os indivíduos com baixo funcionamento da TREM2 - e por consequência baixos níveis de sTREM2 - teriam um processo amiloidogênico mais acelerado, estando super-representados na fase pré-clínica com somente a positividade $A \beta^{33,34}$.

Foi observado no estudo de Banerjee et al. ${ }^{23}$ a falta de variação significante dos níveis de sTREM2 entre controles saudáveis, pacientes de DA e de angiopatia cerebral amiloide ${ }^{23}$. Uma tendência a menores valores de sTREM2 em LCR em pacientes de DAem comparação 
a doença demência com Corpos de Lewy foi encontrada no estudo de Morenas-Rodríguez et al. ${ }^{31}$.

Já foi descrita a associação entre níveis elevados de sTREM2 no LCR e o aumento de volume de massa cinzenta em pacientes com CCL nos córtices temporal medial e inferior bilateral, pré-cúneo e giro supramarginal esquerdo, além de haver redução de difusividade da água no grupo com $\mathrm{CCL}$, sugestivo de edema, podendo se interpretar que o sTREM2LCR está associado a neuroinflamação causada pelo processo inicial de neurodegeneração ${ }^{62}$.

Os níveis de sTREM2 não variam entre portadores e não portadores de variantes raras do gene Trem2 segundo Suárez-Calvet et al. ${ }^{34}$, entretanto, os autores observaram um aumento de sTREM2-LCR na p.R47H e p.H157Y, diminuição na p.L211P, e valores iguais na p.R62H. Também não há diferença nas concentrações de sTREM2 em LCR entre portadores e não portadores de APOE $\varepsilon 4^{18,36,28,41}$. Foi demonstrado que a variante de risco rs7232 do gene MS4A6A está associada aos níveis de sTREM2 ${ }^{36}$.

Foi descrito o aumento do RNAm de TREM2 em sangue periférico em relação a controles e $\mathrm{CCL}^{36,40}$, estando associado a pior memoria episódica, ao déficit cognitivo e a atrofia do hipocampo ${ }^{40}$, sendo assim a expressão de TREM2 mais uma forma de acompanhar o desenvolvimento da doença. O estudo de Hu et al. ${ }^{42}$ demonstrou o aumento da concentração de RNAm em sangue periférico com um AUC de 0,596 para diferenciar DA de controles, e o aumento da concentração proteica em monócitos, com AUC de 0,748 , acurácia diagnóstica de $70 \%$, sensitividade de $68 \%$ e especificidade de $72 \%$, além de sua correlação com a pontuação no miniexame de estado mental ${ }^{42}$. Foi vista também a tendência de up-regulation de TREM2 em granulócitos e em plasma, sugerindo que a diferença de expressão de RNAm de TREM2 venha principalmente de monócitos ${ }^{53}$. Nos estudos de Mori et al. ${ }^{55}$ e Casati et al..$^{43}$ foi visto o aumento do RNAm de TREM2 em leucócitos de pacientes de DA, e no estudo de Ozaki et al. ${ }^{30}$ observou-se essa elevação nos leucócitos de portadores de APOE $\varepsilon 4$ em comparação a de não portadores, mas somente em indivíduos com CCL que avançariam para DA, e não em CCL sem características de progressão para DA. O aumento da expressão de TREM2 pode refletir um mecanismo compensatório da micróglia em relação a deposição amiloide e o aumento da demanda fagocítica devido à patologia neurítica $e$ células apoptóticas, e dado que a DA continua a progredir, a superexpressão de TREM2 pode ser uma tentativa insuficiente de reparar o tecido cerebral ${ }^{40}$.

\section{GFAP}

A proteína acídica fibrilar glial (GFAP) é uma proteína do citoesqueleto dos astrócitos, que é liberada durante o processo de neurodegenerativo da DA e considerada um marcador de astrogliose ${ }^{37,38}$. A expressão da GFAP está aumentada na DA em relação a controles e a doença de Parkinson, estando associada ao dano a substância branca por meio de sua correlação negativa com a densidade de mielina, sendo este um fenômeno ligado a disfunção cognitiva e fator importante na etiologia da $\mathrm{DA}^{47}$. $\mathrm{O}$ estudo de Milà-alomà et al..$^{59}$ demonstrou o aumento do GFAP ao longo da idade em indivíduos tanto $A \beta$-negativos quanto positivos na pré-clínica, porém está mais pronunciado nos $A \beta$-positivo ${ }^{53}$. Em indivíduos sintomáticos (CCLe demência) com perfil positivo $\mathrm{DA}$ (definido pela razão p-tau/Aß42), os níveis de GFAP mostraram-se mais elevados em relação aos de perfil negativo ${ }^{18}$.

Foi demonstrado o aumento de GFAP no LCR em pacientes de DA em relação a controles ${ }^{22,52}$, mas também em pacientes com doença de príon, $\mathrm{DFT}^{20}$, demência com corpo de Lewy (DCL) e degeneração lobular frontotemporal (DLFT) ${ }^{38}$, sem diferença significativa entre as doenças, com exceção a DLFT, onde os níveis foram mais elevados ${ }^{38}$, demonstrando pouca utilidade para o diagnóstico diferencial. O mesmo foi observado no GFAP sérico no estudo de Benussi et al. ${ }^{53}$, sem diferenças significantes entre as concentrações em DA e qualquer subgrupo de DLFT.

GFAP em LCR apresentou baixa capacidade de distinção $(A \cup C=0,64)$ entre indivíduos com perfil de $D A$ e não-DA determinado pela razão t-tau/A $\beta 42^{61}$. Por outro lado, foi demonstrado por Oeckl et al. ${ }^{37} \mathrm{com}$ imunoensaio na plataforma Single molecule array (Simoa) que o GFAP sérico é capaz de distinguir DA não só de controles $(A \cup C=0,91)$ - sendo superior ao valor encontrado para $A \beta 42(0,87)$ - mas também de indivíduos com a variante comportamental de DFT (AUC $=0,85)$, e apresentando concentração duas vezes maior que na doença de Parkinson, além de apresentar uma correlação com comprometimento cognitivo ${ }^{37}$. O estudo de Asken et al. ${ }^{48}$ identificou um crescimento linear em adultos mais velhos com leves alterações de cognição ou com cognição intacta. Contudo, ainda no mesmo estudo, em outra coorte que incluía indivíduos com demência e maior acúmulo amiloide, foi observada uma relação curvilinear entre a concentração plasmática de GFAP e a deposição amiloide, mensurada por PET scan em Centiloids (CLs), foi visto um aumento de GFAP até em torno dos 75 CLs antes de entrar em platô e então diminuir a partir dos $100 \mathrm{CLs}$, havendo forte associação negativa entre GFAP e A $\beta-P E T$ em indivíduos com demência ${ }^{48}$. Esse padrão pode representar a utilidade de GFAP como um marcador precoce de neurodegeneração, além de que diferentes níveis de GFAP poderiam sinalizar diferentes estágios do desenvolvimento da DA.

\section{S100B}

A proteína B S100 ligante de cálcio (S100B) é uma das proteínas solúveis mais abundantes no 
cérebro. Ela é expressa primariamente em astrócitos e encontrasse aumentada na DA ${ }^{61,18,57}$, mas também em outras doenças, como a demência vascular, como demonstrado por Llorens et al. ${ }^{57}$, não havendo diferença significante de S100B entre ambas, e o marcadores sendo incapaz de distingui-las ${ }^{57}$. No estudo de Van Hulle et al. ${ }^{18}$, os níveis de S100B em LCR se mostrou mais alto no grupo com perfil de DA (determinado pela razão $p$-tau/A $\beta 42$ ) em indivíduos com comprometimento cognitivo (CCL e demência), enquanto não houve diferença em indivíduos sem comprometimento cognitivo ${ }^{18}$. O mesmo estudo apresentou fraca correlação de S100B com t-tau e neurofilamento leve ${ }^{18}$, contudo, no trabalho de Teitsdottir et al. ${ }^{61}$, foi demonstrada correlação mais forte de S100B com esses marcadores, mas não com Aß42. Também no estudo de Teitsdottir et al. ${ }^{61}$ foi demonstrada má capacidade de S100B em LCR para diferenciar indivíduos com perfil de DA dos não-DA ( $A \cup C=0,61)$, definido pela razão t-tau/ $A \beta 42^{61}$. No soro foi visto um aumento considerável dos níveis de S100B em pacientes de DA, entre os que apresentavam a demência a até 5 anos e considerados com demência moderada houve um aumento de 60 vezes em comparação aos controles ${ }^{32}$.
Curiosamente, no grupo com mais de 10 anos de duração da doença, os indivíduos com demência moderada apresentaram um aumento apenas de 10 vezes, e os com demência severa 37 vezes $^{32}$.

\section{CONCLUSÃO}

No geral os marcadores gliais não se mostram eficazes para o diagnóstico diferencial, mas mostram bom potencial para atuação no prognostico, além de poderem ter utilidade como biomarcadores inespecíficos para a neurodegeneração já nos estágios iniciais do desenvolvimento patológico. GFAP em especial pode ser um bom candidato como marcador sanguíneo, demonstrando capacidade diagnóstica comparável a $A \beta 42$ e tau em LCR, e com a capacidade de distinguir de DFT e doença de Parkinson, de acordo com o estudo de Oeckl et al. ${ }^{37}$. Há, contudo, uma escassez de estudos sobre GFAP para corroborar esses resultados, sendo necessários mais estudos para validá-los. TREM2 é uma alternativa sanguínea com seu RNAm ou mesmo a própria proteína em leucócitos periféricos. O potencial efeito modulatório/ protetivo da ativação glial pode representar um alvo para intervenções terapêuticas.

\section{REFERÊNCIAS}

1. Muszynski P, Groblewska M, Kulczynska-Przybik A, Kułakowska A, Mroczko B. YKL-40 as a potential biomarker and a possible target in therapeutic strategies of Alzheimer's disease. Curr Neuropharmacol. 2017;15(6):906-17.

2. Blennow K, Dubois B, Fagan AM, Lewczuk P, Leon MJ, Hampel $\mathrm{H}$. Clinical utility of cerebrospinal fluid biomarkers in the diagnosis of early Alzheimer's disease. Alzheimers Dement. 2015;11(1):58-69.

3. Machado APR, Carvalho IO, Rocha Sobrinho HM. Neuroinflamação na doença de Alzheimer. Rev Bras Mil Ciencias. 2020;6(14).

4. Lane CA, Hardy J, Schott JM. Alzheimer's disease. Eur J Neurol. 2018;25(1):59-70.

5. Molinuevo JL, Ayton S, Batrla R, Bednar MM, Bittner T, Cummings J. Current state of Alzheimer's fluid biomarkers. Acta Neuropathol. 2018;136(6):821-53.

6. Vergallo A, Lista S, Lemercier P, Chiesa PA, Zetterberg $\mathrm{H}$, Blennow K, et al. Association of plasma YKL-40 with brain amyloid-b levels, memory performance, and sex in subjective memory complainers. Neurobiol Aging. 2020;96:22-32.

7. Nordengen K, Kirsebom B, Henjum K, Selnes P, Gisladottir B, Wettergreen M, et al. Glial activation and inflammation along the Alzheimer's disease continuum. J Neuroinflammation. 2019;16(1):46.

8. Colangelo AM, Alberghina L, Papa M. Astrogliosis as a therapeutic target for neurodegenerative diseases. Neurosci Lett. 2014;565:59-64.

9. Sutphen $C L$, Jasielec MS, Shah AR, Macy EM, Xiong C, Vlassenko AG, et al. Longitudinal cerebrospinal fluid biomarker changes in preclinical alzheimer disease during middle age. JAMA Neurol. 2015;72(9):1029-42.

10. Craig-Schapiro R, Perrin RJ, Roe $\mathrm{CM}$, Xiong C, Carter D, Cairns NJ, et al. YKL-40: a novel prognostic fluid biomarker for preclinical Alzheimer's disease. Biol Psychiatry. 2010;68(10):903-12.

11. Paterson RW, Heywood WE, Heslegrave AJ, Magdalinou NK, Andreasson U, Sirka E, et al. A targeted proteomic multiplex
CSF assay identifies increased malate dehydrogenase and other neurodegenerative biomarkers in individuals with Alzheimer's disease pathology. Transl Psychiatric. 2016;6(11):e952.

12. Hampel $\mathrm{H}$, Toschi $\mathrm{N}$, Baldacci $\mathrm{F}$, Zetterberg H, Blennow K, Kilimann I, et al. Alzheimer's disease biomarkerguided diagnostic workflow using the added value of six combined cerebrospinal fluid candidates: A $\beta 1-$ 42, total-tau, phosphorylated-tau, NFL, neurogranin, and YKL-40. Alzheimer Dement. 2018;14(4):492-510.

13. Alcolea D, Vilaplana E, Suarez-Calvet M, Illan-Gala I, Blesa R, Clarimon J, et al. CSF SAPP $\beta$, YKL-40, and neurofilament light in frontotemporal lobar degeneration. Neurology. 2017;89(2):178-88.

14. Paterson RW, Slattery CF, Poole T, Nicholas JM, Magdalinou NK, Toombs $\mathrm{J}$, et al. Cerebrospinal fluid in the differential diagnosis of Alzheimer's disease: clinical utility of an extended panel of biomarkers in a specialist cognitive clinic. Alzheimers Res Ther. 2018;10(1):32. 
15. Mattsson N, Tabatabaei S, Johansson $\mathrm{P}$, Hansson $\mathrm{O}$, Andreasson U, Mansson JE, et al. Cerebrospinal fluid microglial markers in Alzheimer's disease: elevated chitotriosidase activity but lack of diagnostic utility. Neuromolecular Med. 2011;13(2):151-9.

16. Antonell A, Tort-Merino A, Rios J, Balasa M, Borrego-Ecija S, Auge $\mathrm{JM}$, et al. Synaptic, axonal damage and inflammatory cerebrospinal fluid biomarkers in neurodegenerative dementias. Alzheimers Dement. 2020;16(2)262-72.

17. Baldacci F, Toschi N, Lista S, Zetterberg $\mathrm{H}$, Blennow K, Kilimann I, et al. Two-level diagnostic classification using cerebrospinal fluid YKL-40 in Alzheimer's disease. Alzheimers Dement. 2017;13(9)993-1003.

18. Van Hulle C, Jonaitis EM, Betthauser TJ, Batrla R, Wild N, Kollmorgen $\mathrm{G}$, et al. An examination of a novel multipanel of CSF biomarkers in the Alzheimer's disease clinical and pathological continuum. Alzheimers Dement. 2021;17(3):431-45.

19. Gispert JD, Monte GC, Falcon C, Tucholka A, Rojas S, Sanchez-Valle R, et al. CSF YKL-40 and pTau181 are related to different cerebral morphometric patterns in early $A D$. Neurobiol Aging. 2016;38:47-55.

20. Abu-Rumeileh $S$, Steinacker $P$, Polischi B, Mammana A, BartolettiStella A, Oeckl P, et al. CSF biomarkers of neuroinflammation in distinct forms and subtypes of neurodegenerative dementia. Alzheimers Res Ther. 2019;12(1):2.

21. Rauchmann BS, Schneider-Axmann T, Alexopoulos P, Perneczky R, Alzheimer's Disease Neuroimaging Initiative. CSF soluble TREM2 as a measure of immune response along the Alzheimer's disease continuum. Neurobiol Aging. 2019;74:182-90.

22. Lauridsen C, Sando SB, Moller I, Berge G, Pomary PK, Grontvedt $G R$, et al. Cerebrospinal fluid $A \beta 43$ is reduced in early-onset compared to late-onset Alzheimer's disease, but has similar diagnostic accuracy to A 342 . Front Aging Neurosci. 2017;9:210.

23. Banerjee G, Ambler G, Keshavan A, Paterson RW, Foiani MS, Toombs J, et al. Cerebrospinal fluid biomarkers in cerebral amyloid angiopathy. $J$ Alzheimers Dis. 2020;74(4):1189-201.
24. Llorens F, Thune K, Tahir W, Kanata E, Diaz-Lucena D, Xanthopoulos K, et al. YKL-40 in the brain and cerebrospinal fluid of neurodegenerative dementias. Mol Neurodegener. 2017;12(1):83.

25. Bos I, Vos S, Verhey F, Scheltens P, Teunissen C, Engelborghs S, et al. Cerebrospinal fluid biomarkers of neurodegeneration, synaptic integrity, and astroglial activation across the clinical Alzheimer's disease spectrum. Alzheimer's Dement. 2019;15(5): 644-54.

26. Knapskog AB, Henjum K, Idland AV, Eldholm RS, Persson K, Saltvedt I, et al. Cerebrospinal fluid sTREM2 in Alzheimer's disease: comparisons between clinical presentation and AT classification. Sci Rep. 2020;10(1):15889.

27. Henjum K, Almdahl IS, Arskog V, Minthon L, Hansson O, Fladby T, et al. Cerebrospinal fluid soluble TREM2 in aging and Alzheimer's disease. Alzheimers Res Ther. 2016;8(1):17.

28. Brosseron F, Traschutz A, Widmann CN, Kummer MP, Tacik P, Santarelli $F$, et al. Characterization and clinical use of inflammatory cerebrospinal fluid protein markers in Alzheimer's disease. Alzheimers Res Ther. 2018;10(1):25.

29. Deming $Y$, Black $K$, Carrell $D$, Cai $Y$, Del-Aguila JL, Fernandez MV, et al. Chitinase-3-like 1 protein (CHI3L1) locus influences cerebrospinal fluid levels of YKL-40. BMC Neurol. 2016;16(1):217.

30. Ozaki Y, Yoshino Y, Yamazaki K, Sao T, Mori Y, Ochi S, et al. DNA methylation changes at TREM2 intron 1 and TREM 2 mRNA expression in patients with Alzheimer's disease. J Psychiatr Res. 2017;92:74-80.

31. Morenas-Rodriguez E, Alcolea $D$, Suarez-Calvet M, Munoz-Llahuna L, Vilaplana E, Sala I, et al. Different pattern of CSF glial markers between dementia with Lewy bodies and Alzheimer's disease. Sci Rep. 2019;9:1-10.

32. Gruden MA, Davidova TB, Malisauskas M, Sewell RDE, Voskresenskaya NI, Wilhelm K, et al. Differential neuroimmune markers to the onset of Alzheimer's disease neurodegeneration and dementia: autoantibodies to
$A \beta(25-35)$ oligomers, S100b and neurotransmitters. J Neuroimmunol. 2007;186(1-2):181-92.

33. Ma LZ, Tan L, Bi YL, Shen XN, Xu $\mathrm{W}, \mathrm{Ma} Y \mathrm{YH}$, et al. Dynamic changes of CSF STREM2 in preclinical Alzheimer's disease: the CABLE study. Mol Neurodegener. 2020;15:19.

34. Suarez-Calvet M, Morenas-Rodriguez E, Kleinberger G, Schlepckow K, Angel M, Caballero MAA, et al. Early increase of CSF sTREM2 in Alzheimer's disease is associated with tau related- neurodegeneration but not with amyloid- $\beta$ pathology. Mol Neurodegener. 2019;14(1):1.

35. Liu C, Yu J. Genome-Wide Association Studies for Cerebrospinal Fluid Soluble TREM2 in Alzheimer's disease. Front Aging Neurosci. 2019;11:297.

36. Hou XH, Bi YL, Tan MS, Xu W, Li JQ, Shen XN, et al. Genomewide association study identifies Alzheimer's risk variant in MS4A6A influencing cerebrospinal fluid sTREM2 levels. Neurobiol Aging. 2019;84:241.e13-20.

37. Oeckl P, Halbgebauer S, AnderlStraub S, Steinacker P, Huss AM, Neugebauer $\mathrm{H}$, et al. Glial fibrillary acidic protein in serum is increased in Alzheimer's disease and correlates with cognitive impairment. $J$ Alzheimers Dis. 2018;67(2):481-8

38. Ishiki A, Kamada M, Kawamura Y, Terao C, Shimoda F, Tomita N, et al. Glial fibrillar acidic protein in the cerebrospinal fluid of Alzheimer's disease, dementia with Lewy bodies, and frontotemporal lobar degeneration. $J$ Neurochem. 2016;136(2):258-61.

39. Ewers M, Biechele G, Suarez-Calvet M, Sacher C, Blume T, MorenasRodriguez E, et al. Higher CSF sTREM 2 and microglia activation are associated with slower rates of betaamyloid accumulation. EMBO Mol Med. 2020;12(9):e12308.

40. Tan JY, Ng ASL, Vipin A, Lim JKW, Chander RJ, Ji F, et al. Higher peripheral TREM2 mRNA levels relate to cognitive deficits and hippocampal atrophy in Alzheimer's disease and amnestic mild cognitive impairment. J Alzheimers Dis. 2017;58(2):413-23. 
41. Heslegrave A, Heywood W, Paterson R, Magdalinou N, Svensson J, Johansson P, et al. Increased cerebrospinal fluid soluble TREM2 concentration in Alzheimer's disease. Mol Neurodegener. 2016;11:3.

42. Hu N, Tan MS, Yu JT, Sun L, Tan L, Wang $Y L$, et al. Increased expression of TREM2 in peripheral blood of Alzheimer's disease patients. $J$ Alzheimers Dis. 2014;38(3);497-501.

43. Casati M, Ferri E, Gussago C, Mazzola P, Abbate C, Bellelli G, et al. Increased expression of TREM2 in peripheral cells from mild cognitive impairment patients who progress into Alzheimer's disease. Eur J Neurol. 2018;25(6):805-10.

44. Lleo A, Alcolea D, Martinez-Lage P, Scheltens P, Parnetti L, Poirier J, et al. Longitudinal cerebrospinal fluid biomarker trajectories along the Alzheimer's disease continuum in the BIOMARKAPD study. Alzheimers Dement. 2019;15(6):742-53.

45. Sutphen CL, McCue L, Herries EM, Xiong $\mathrm{C}$, Landerson $\mathrm{JH}$, Holtzman DM, et al. Longitudinal decreases in multiple cerebrospinal fluid biomarkers of neuronal injury in symptomatic late onset Alzheimer's disease. Alzheimer Dement. 2018;14(7):869-79.

46. Merluzzi AP, Carlsson CM, Johnson SC, Schindler SE, Asthana S, Blennow K, et al. Neurodegeneration, synaptic dysfunction, and gliosis are phenotypic of Alzheimer dementia. Neurology. 2018;91(5):e436-43.

47. Han F, Perrin RJ, Wang Q, Wang Y, Perlmutter JS, Morris JC, et al. Neuroinflammation and myelin status in Alzheimer's disease, Parkinson's disease, and normal aging brains: a small sample study. Parkinsons Dis. 2019:7975407.

48. Asken BM, Elahi FM, La Joie R, Strom A, Staffaroni AM, Lindbergh $\mathrm{CA}$, et al. Plasma glial fibrillary acidic protein levels differ along the spectra of amyloid burden and clinical disease stage. J Alzheimers Dis. 2020;78(1):265-76.
49. Villar-Pique A, Schmitz M, Hermann P, Goebel S, Bunck T, Varges D, et al. Plasma YKL-40 in the spectrum of neurodegenerative dementia. $J$ Neuroinflammation. 2019;16(1):145.

50. Choi J, Lee HW, Suk K. Plasma level of chitinase 3-like 1 protein increases in patients with early Alzheimer's disease. J Neurol. 2011;258(12):2181-5.

51. Morenas-Rodriguez E, CerveraCarles L, Vilaplana E, Alcolea D, Carmona-Iraguia M, Dols-Icardo $\mathrm{O}$, et al. Progranulin protein levels in cerebrospinal fluid in primary neurodegenerative dementias. $J$ Alzheimers Dis. 2016;50(2):539-46.

52. Sathe $\mathrm{G}, \mathrm{Na} \mathrm{CH}$, Renuse $\mathrm{S}$, Madugundu AK, Moghekar A, Pandey A. Quantitative proteomic profiling of cerebrospinal fluid to identify candidate biomarkers for Alzheimer's disease. Proteomics Clin Appl. 2019;13(4) :e1800105.

53. Benussi A, Ashton NJ, Karikari TK, Gazzina S, Premi E, Benussi L, et al. Serum glial fibrillary acidic protein (GFAP) is a marker of disease severity in frontotemporal lobar degeneration. J Alzheimers Dis. 2020;77(3):1129-41.

54. Rauchmann BS, Sadlon A, Perneczky R, Alzheimer's Disease Neuroimaging Initiative. Soluble TREM2 and inflammatory proteins in Alzheimer's disease cerebrospinal fluid. $J$ Alzheimers Dis. 2020;73(4):165-26.

55. Mori Y, Yoshino Y, Ochi S, Yamazaki $\mathrm{K}$, Kawabe K, Abe M, et al. TREM2 mRNA Expression in leukocytes is increased in Alzheimer's disease and schizophrenia. PLoS One. 2015;10(9):e0136835.

56. Gispert JD, Mont GC, Suarez-Calvet M, Falcon C, Tucholka A, Rojas S, et al. The APOE \&4 genotype modulates CSF YKL-40 levels and their structural brain correlates in the continuum of Alzheimer's disease but not those of sTREM2. Alzheimers Dement. 2017;6:50-9.

57. Llorens F, Schmitz M, Knipper T, Schmidt C, Lange P, Fischer A, et al. Cerebrospinal fluid biomarkers of Alzheimer's disease show different but partially overlapping profile compared to vascular dementia. Front Aging Neurosci. 2017;9:289.
58. Suarez-Calvet $M$, Kleinberger G, Caballero MAA, Brendel M, Rominger A, Alcolea D, et al. sTREM 2 cerebrospinal fluid levels are a potential biomarker for microglia activity in early-stage Alzheimer's disease and associate with neuronal injury markers. EMBO Mol Med. 2016;8(5):466-76.

59. Mila-Aloma M, Salvado G, Gispert JD, Vilor-Tejedor N, Grau-Rivera, Sala-Vila A, Crous-bou M, et al. Amyloid beta, tau, synaptic, neurodegeneration, and glial biomarkers in the preclinical stage of the Alzheimer's continuum. Alzheimers Dement. 2020;16(10):1358-71.

60. Alcolea $D$, Martinez-Lage $P$, Sanchez-Juan P, Olazaran J, Antunez C, Izagirre A, et al. Amyloid precursor protein metabolism and inflammation markers in preclinical Alzheimer disease. Neurology. 2015;85(7):626-33.

61. Teitsdottir UD, Jonsdottir MK, Lund $\mathrm{SH}$, Darreh-Shori T, Snaedal J, Petersen PH. Association of glial and neuronal degeneration markers with Alzheimer's disease cerebrospinal fluid profile and cognitive functions. Alzheimers Res Ther. 2020;12(1):92.

62. Suarez-Calvet M, Capell A, Caballero MAA, Morenas-Rodriguez $\mathrm{E}$, Fellerer K, Franzmeier N, et al. CSF progranulin increases in the course of Alzheimer's disease and is associated with STREM 2, neurodegeneration and cognitive decline. EMBO Mol Med. 2018;10(12):e9712.

63. Palmqvist $S$, Insel PS, Stomrud E, Janelidze S, Zetterberg H, Brix $B$, et al. Cerebrospinal fluid and plasma biomarker trajectories with increasing amyloid deposition in Alzheimer's disease. EMBO Mol Med. 2019;11(12):1-13.

64. Wang L, Gao T, Cai T, Li K, Zheng P. Cerebrospinal fluid levels of YKL-40 in prodromal Alzheimer's disease. Neurosci Lett. 2020;715:134658.

65. Hoglund K, Kern S, Zettergren A, Borjesson-Hansson A, Zetterberg $H$, Skoog I, et al. Preclinical amyloid pathology biomarker positivity: effects on tau pathology and neurodegeneration. Transl Psychiatry. 2017;7(1):e995. 